\title{
Hospital acquired vancomycin resistant enterococci in surgical intensive care patients - a prospective longitudinal study
}

\author{
Stefanie Kampmeier ${ }^{1 *+} \mathbb{D}$, Annelene Kossow ${ }^{1 \dagger}$, Larissa Monika Clausen ${ }^{1}$, Dennis Knaack ${ }^{2}$, Christian Ertmer $^{3}$,
} Antje Gottschalk ${ }^{3}$, Hendrik Freise ${ }^{3}$ and Alexander Mellmann ${ }^{1}$

\begin{abstract}
Background: Vancomycin resistant enterococci (VRE) occur with enhanced frequency in hospitalised patients. This study elucidates the prevalence of VRE on admission among surgical intensive care unit (SICU) patients, whether these patients are at special risk for VRE acquisition and which risk factors support this process.

Methods: Patients admitted to SICUs of the University Hospital Münster were examined during August-October 2017. VRE screening was performed within $48 \mathrm{~h}$ after admission and directly prior to discharge of patients. In parallel risk factors were recorded to estimate their effect on VRE acquisition during SICU stay.

Results: In total, 374 patients (68\% male) with a median age of 66 years were admitted to one of the SICUs during the investigation period. Of all, 336 patients (89.8\%) were screened on admission and 268 (71.7\%) on discharge. Nine patients were admitted with previously known VRE colonisation. Twelve (3.6\%) further patients were VRE positive on admission. During ICU stay, eight (3.0\%) additional patients turned out to be VRE colonised. Risk factors found to be significantly associated with VRE acquisition were median length of stay on the ICU (14 vs. 3 days; $p=$ 0.01 ), long-term dialysis ( $12.5 \%$ vs. $2.0 \%$ of patients; $p=0.05$ ), and antibiotic treatment with flucloxacillin ( $28.6 \%$ vs. 7. $2 \%$ of patients; $p=0.01$ ) or piperacillin/tazobactam ( $57.1 \%$ vs. $26.6 \%$ of patients; $p=0.01$ ).

Conclusions: SICU patients are not at special risk for VRE acquisition. Previous stay on a SICU should therefore not be considered as specific risk factor for VRE colonisation.
\end{abstract}

Keywords: Vancomycin resistant enterococci, Hospital-acquisition, Risk factors, Surgical intensive care patients

\section{Background}

Enterococci are an emerging pathogen in hospitalised patients [1]. These pathogens ubiquitously occur in the hospital environment and show a high tenacity on inanimate surfaces [2-4]. As a result, enterococcal infections emerge with a rising frequency. Additionally, enterococci have the ability of acquiring resistances to multiple antimicrobial agents and the capacity to transfer resistances to other pathogens via mobile genetic elements [5-7]. For this reason the prevalence of vancomycin resistant enterococci (VRE) has increased intensively [1]. Vancomycin resistance

\footnotetext{
* Correspondence: Stefanie.Kampmeier@ukmuenster.de

${ }^{\dagger}$ Stefanie Kampmeier and Annelene Kossow contributed equally to this work. ${ }^{1}$ Institute of Hygiene, University Hospital Münster, Robert-Koch-Strasse 41, 48149 Münster, Germany

Full list of author information is available at the end of the article
}

is associated with enhanced mortality, e.g. among patients with enterococcal blood stream infections [8]. Within hospital settings prevention of VRE transmission is therefore a major objective.

Infection control strategies to control VRE vary, depending on local guidelines. Usually bundle strategies are applied to prevent transmission of VRE between two patients [9]. These include contact precautions, intensified disinfection strategies, the usage of personal protective equipment and active surveillance $[10,11]$. In this context, screening strategies are controversially discussed [12]. Both, generalised screening and a risk-adaptive screening, are possible approaches. Regarding the latter, the question arises, which patient groups should be included. Acquisition of VRE in critical ill patients has been associated with prolonged duration of hospital stay, previous hospitalisations, antibiotic

(c) The Author(s). 2018 Open Access This article is distributed under the terms of the Creative Commons Attribution 4.0 International License (http://creativecommons.org/licenses/by/4.0/), which permits unrestricted use, distribution, and reproduction in any medium, provided you give appropriate credit to the original author(s) and the source, provide a link to the Creative Commons license, and indicate if changes were made. The Creative Commons Public Domain Dedication waiver (http://creativecommons.org/publicdomain/zero/1.0/) applies to the data made available in this article, unless otherwise stated. 
treatment, long-term dialysis and immunosuppression [13-15]. Surgical intensive care patients host a variety of these risk factors, although not explicitly mentioned as risk clientele. Whether surgical intensive care patients per se are at a special risk to acquire VRE and should therefore be included in a risk adapted screening upon subsequent admission to a hospital has not yet been investigated. The present work addresses these questions and investigates risk factors making a VRE acquisition more probable.

\section{Methods}

\section{Clinical setting and infection control measures}

The 1500-bed University Hospital Münster comprises four interdisciplinary SICUs, hosting abdominal-, trauma-, neuro-, vascular- and thoracic-surgery patients. In total, capacity of all ICUs is 43 beds at its maximum distributed over 26 patient rooms.

Routinely, screening for multidrug-resistant organisms includes an admission-screening for methicillin-resistant Staphylococcus aureus (MRSA) and multidrug-resistant Gram-negative bacteria according to the national German guidelines is established [16, 17]. After coincidental detection of VRE in screening swabs and clinical samples of SICU patients, a prospective investigation was initiated, concentrating on VRE acquisition during a stay on a SICU.

Extended hygiene measures in case of VRE detection include contact isolation in a separate room; cohorting of multiple VRE patients is possible. Sanitary facilities are strictly separated and staff is instructed to wear personal protective equipment in case of entering a patient room, consisting of gloves, and gowns. Surface cleaning disinfection was performed once a day.

\section{Detection of risk factors for VRE acquisition}

During the 2-month study period (August - October 2017), risk factors for VRE acquisition were prospectively recorded during the entire in-patient stay. These risk factors included demographic data (age, gender), the duration of stay on the respective SICU, underlying diseases (haemato-oncological, immunosuppressive diseases [e.g. autoimmune-disease, malignancies, HIV-infection, immunomodulatory drug treatment], hepatic insufficiency, liver transplantation, renal insufficiency, long-term dialysis) and medication (systemic glucocorticoid and antibiotic treatment) attributed to VRE acquisition and previous contacts to the healthcare system (admission from a foreign or domestic hospital, admission from an (in-house) ICU) $[13-15,18]$. Patients with a pre-existing VRE status were not included in the analysis, in order to detect risk factors of VRE acquisition on the SICU ward.
VRE screening, culture, antibiotic resistances, PCR testing methods

VRE screening was performed upon patients' admission on the SICU as well as upon discharge of patients from the SICU in order to detect VRE acquisition during SICU stay. Hospital-acquired VRE was defined as acquisition > $48 \mathrm{~h}$ after hospitalisation on the SICU. All VRE not acquired during SICU stay were defined as pre-existing VRE. Swabs were obtained rectally $(5 \mathrm{~cm} a b$ ano) (Transwab $^{\circ} \mathrm{m} 40$ compliant, mwe, Corsham, Wiltshire, UK) and subsequently streaked onto chromogenic selective agar (VRESelect ${ }^{\mathrm{m}}$, Biorad, Hercules, California, USA). Suspected colonies were confirmed via MALDI-TOF-MS (Bruker Corporation, Bremen, Germany). Susceptibility testing was performed in accordance with the current European Committee on Antimicrobial Susceptibility Testing (EUCAST) standards for clinical breakpoints (version 7.0) using the VITEK ${ }^{\circ} 2$ system (BioMérieux, Nürtingen, Germany). Vancomycin resistance was confirmed by the detection of $v a n A, v a n B, v a n C 1$ and $v a n C 2 / 3$ using the GenoType Enterococcus system (Hain Lifescience, Nehren, Germany). Additionally, subsequent whole genome sequence-based typing confirmed presence of van-genes in these isolates.

\section{Whole genome sequence-based typing}

In order to elucidate the clonal relationship of VRE -strains, isolates were compared genetically via whole genome sequencing (WGS) using the Illumina MiSeq platform (Illumina Inc., San Diego, USA) and laboratory procedures as described previously [19]. Coding regions were compared in a gene-by-gene approach (core genome multilocus sequence typing, cgMLST) using the SeqSphere+ software version 4.1 (Ridom GmbH, Münster, Germany) [20]. To visualize the clonal relationship a minimum-spanning tree was generated using the same software. Genotypes that differed in $\leq 3$ cgMLST targets were rated as closely related and highly suspected for a hospital-acquired transmission. For backwards compatibility with classical molecular typing, i. e. MLST, the MLST sequence types (ST) were extracted from the WGS data in silico.

\section{Statistical analysis}

All data are expressed as absolute numbers or percentage, if not stated otherwise. Independent risk factors were determined in a two-step analysis. First, a univariate analysis was performed, selecting potential risk factors. Chi-Square test was used for categorical and the two-sided student's t-test for comparison of numerical data. Second, a logistic regression was performed to ascertain the independency of risk factors. Statistical significance was declared at $p \leq 0.05$. 


\section{Results}

\section{Screening adherence, acquired VRE, genotypes}

Table 1 shows the detailed results of screening data. Of 374 admitted patients, 336 were screened on admission and 268 on discharge respectively, not considering patients with a confirmed VRE status prior to admission or discharge. In total eight hospital-acquired VRE could be detected. Of all pre-existing VRE (on admission), six presented a $v a n A, 14$ a $v a n B$ and one both genotypes. Of all hospital-acquired VRE, one hosted a $\operatorname{van} A$ and seven a $\operatorname{van} B$ genotype.

\section{Antimicrobial resistance expressions}

In total, 29 VRE isolates of 26 patients (one patient was readmitted twice and one patient once) were tested for antimicrobial resistance expressions. Table 2 gives a detailed overview of tested substances.

\section{Whole genome sequence-based typing and VRE transmission}

Of all 29 isolates 25 were subjected to whole genome sequencing. In patients with repetitive VRE detections and the same antimicrobial resistance expression, only the first isolate was undertaken the sequencing procedure. One isolate was not sequenced. Analysing the MLST ST of all sequenced isolates, ST117 was most prevalent (64.0\%) followed by ST80 (24.0\%) and ST721 (11.0\%) (see also Table 2). A genetical comparison with the help of the cgMLST scheme, based on 1423 genes present in all isolates, revealed six clusters, comprising two, three, five and six isolates respectively (Fig. 1). Two of these clusters contain genotypes of pre-existing isolates and isolates detected in both screenings. Six out of eight VRE isolates $(204,283,291,314,361,372)$ detected in patients on discharge, who were previously tested negative for VRE on admission, are genetically closely related to isolates detected in other patients, suggesting a transmission of one VRE clone on ward in these cases (see also Fig. 2). The other two isolates $(300,302)$ detected on discharge are genetically unrelated to other isolates, which can be attributed to selection of VRE e.g. via antibiotic application in these patients or a false-negative screening result on admission.

\section{Analysis of risk factors in SICU patients}

Risk factors were compared between the group of hospital-acquired VRE-patients and all patients being not VRE-colonised or infected hospital-acquired. Patients with a pre-existing VRE status were not included in the analysis, in order to detect risk factors of VRE acquisition on the SICU ward. Risk factors to have a significant influence on VRE acquisition in this patient clientele were found to be long-term dialysis $(p=0.05)$, the median duration of stay $(p=0.01)$, and an antibiotic treatment with flucloxacillin (duration of treatment for 5.3 days $[p=0.01]$ ) or piperacillin/tazobactam (duration of treatment for 4.6 days [ $p=0.01]$ ), while an antibiotic treatment per se did not show a significant risk profile. Further results of the risk factor analyses can be found in Table 3. The multivariate analysis found the duration of stay and flucloxacillin treatment to be statistically significant as shown in Table 4.

\section{Discussion}

Control of VRE is an emerging topic, patients and healthcare workers have to cope with. An important aspect for prevention of transmission is the early recognition of VRE via screening strategies. Here, the question was addressed, whether SICU patients acquire VRE during their stay and which factors put them at special risk for acquisition. VRE acquisition rate $(3.0 \%)$ on the SICU was lower than the initial prevalence $(3.6 \%)$ on admission of all patients. Additionally, no hospital-acquired infection but only colonisations occurred. Distribution of detected van-genotypes and MLST ST (vanB-genotype and ST117 were most prevalent) are thereby in accordance with national results and trends published for German healthcare institutions recently [21]. In contrast to another retrospective study investigating hospital-acquired VRE, where the acquisition rate was $28.6 \%$, on different wards including surgical and internal ICUs and lacking a generalised VRE screening, acquisition of VRE in our investigation was comparably low [22]. Further investigations on haemato-oncological patients revealed one third of these patients to develop hospital-acquired VRE [18]. SICU patients should per se not be considered as a specific risk clientele for VRE acquisition in comparison with these patients.

Table 1 Screening data of SICU-admitted patients $(n=374)$ during August to October 2017

\begin{tabular}{|c|c|c|c|c|c|}
\hline & \multicolumn{5}{|l|}{ Screening result } \\
\hline & \multicolumn{3}{|l|}{ Positive } & \multirow[t]{2}{*}{ Negative } & \multirow[t]{2}{*}{ Total } \\
\hline & vanA genotype & van B genotype & van $A$ and $\operatorname{van} B$ genotype & & \\
\hline Pre-existing VRE & $6(66.7 \%)$ & $3(33.3 \%)$ & $0(0 \%)$ & I & 9 \\
\hline Screening on admission & $0(0 \%)$ & $11(3.3 \%)$ & $1(0.3 \%)$ & $324(96.4 \%)$ & 336 \\
\hline Screening on discharge & $1(0.4 \%)$ & $7(2.6 \%)$ & $0(0 \%)$ & $260(97.0 \%)$ & 268 \\
\hline
\end{tabular}




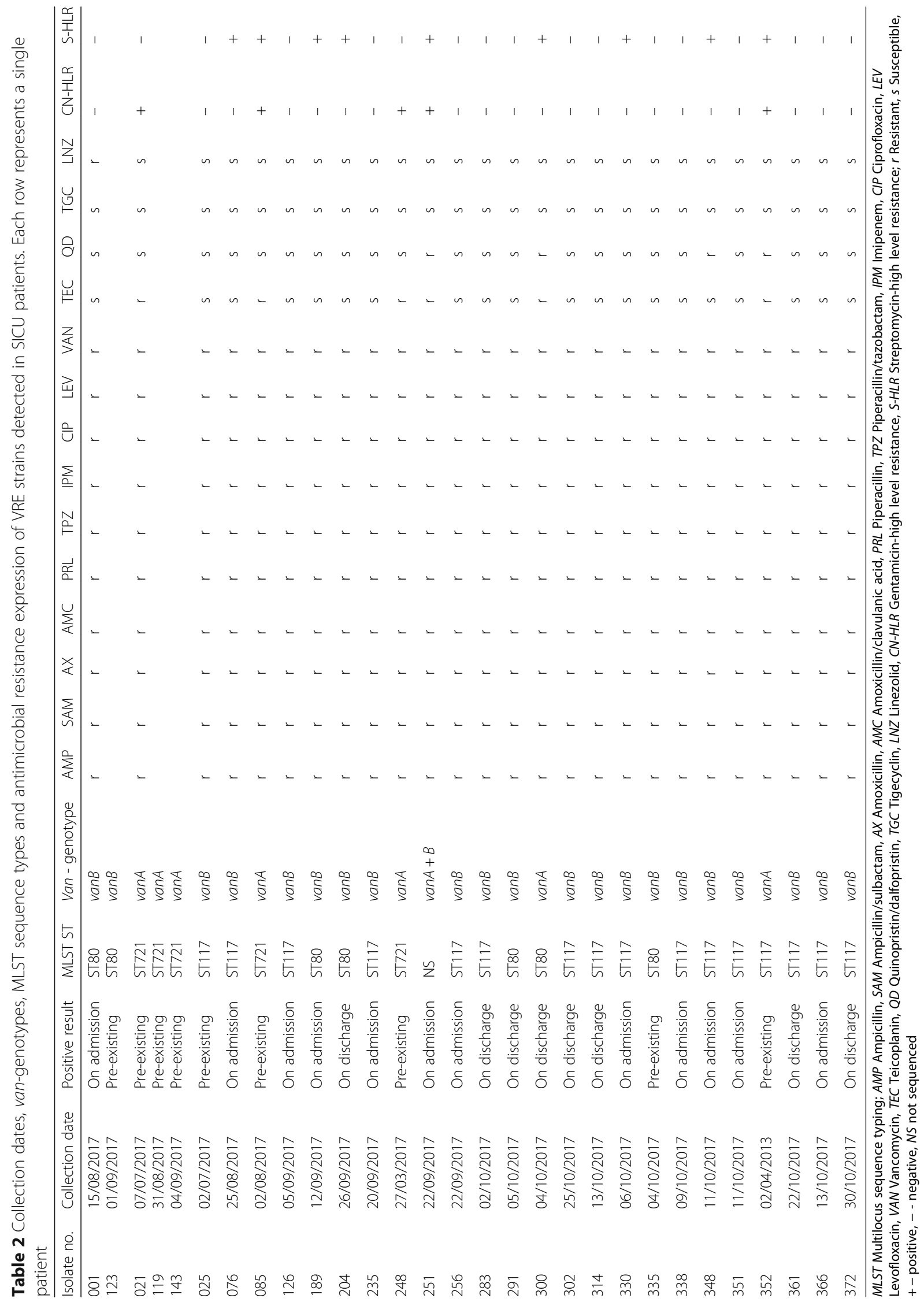




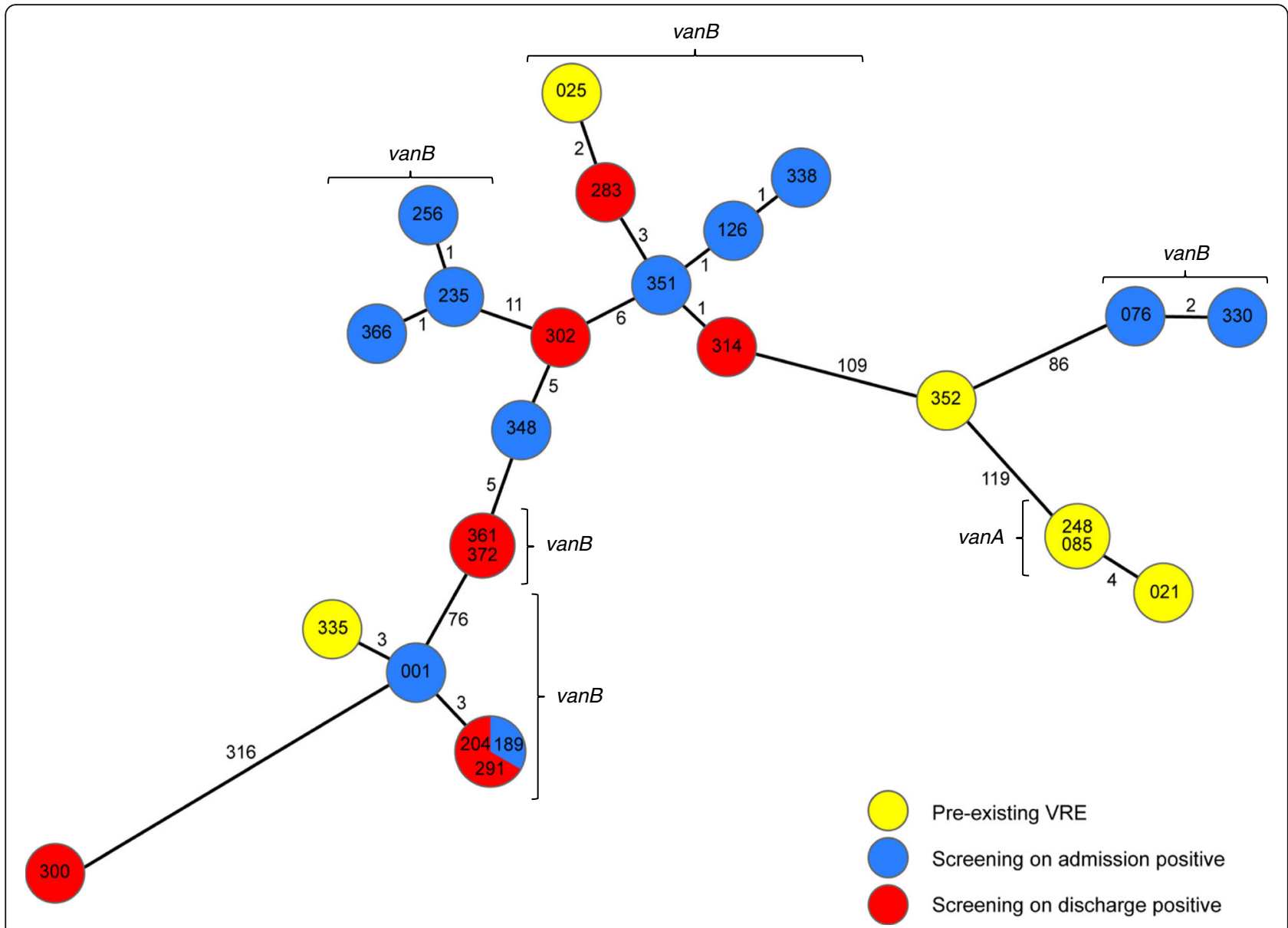

Fig. 1 Minimum spanning tree of VRE isolates illustrating their genotypic relationship. Minimum spanning tree of 25 VRE strains isolated from intensive care unit patients with VRE anamnesis (yellow) and detected during screening on admission (blue) and on discharge (red) during August and October 2017 based on 1423 cgMLST target genes [20], pairwise ignoring missing values. Genotypes are numbered chronologically in order of patients' admission on ICU. Each dot represents one genotype. Size of dots correlates with the number of identical genotypes. Numbers near to the connecting lines show the number of alleles differing between two genotypes. Whole Genome Sequencing revealed six clusters of VRE, one vanA-cluster and five vanB-clusters

Nevertheless, since hospital-acquired VRE colonisations occurred in our patients, risk factors supporting a VRE acquisition in this group of patients were assessed. Here, the median duration of stay, long-term dialysis and the antibiotic treatment with flucloxacillin or piperacillin/tazobactam were found to be the predominant risk factors. These data correspond with previously investigated risk factors and certainly cannot be considered independently but presuppose each other [23, 24], as also verified by the multivariate analysis. Other risk factors that were found to play an important role in VRE acquisition in other patient clientele could not be verified for our SICU patients. Here, the attributed risk of VRE acquisition after cephalosporin treatment needs to be mentioned. Due to perioperative antibiotic prophylaxis standards, especially referring to cardiac surgery patients, approximately $60 \%$ of all admitted patients received cefuroxime; however, this did not lead to an increased VRE acquisition rate. This is noteworthy, since cephalosporine use promotes selection of enterococci and thereby VRE due to intrinsic resistance mechanisms. Nevertheless, previous studies could confirm, that cephalosporine use per se during hospitalization does not result in an enhanced VRE carriage [14]. Interestingly, since the duration of application of flucloxacillin and piperacillin/tazobactam did not vary significantly among the hospital-acquired VRE and the non VRE group, the present results suggest that few antibiotic applications can be sufficient to potentially select VRE. The impact of relatively low numbers of antibiotic administrations on selection of certain pathogens corroborates similar findings for the selection of Clostridium difficile [25]. 


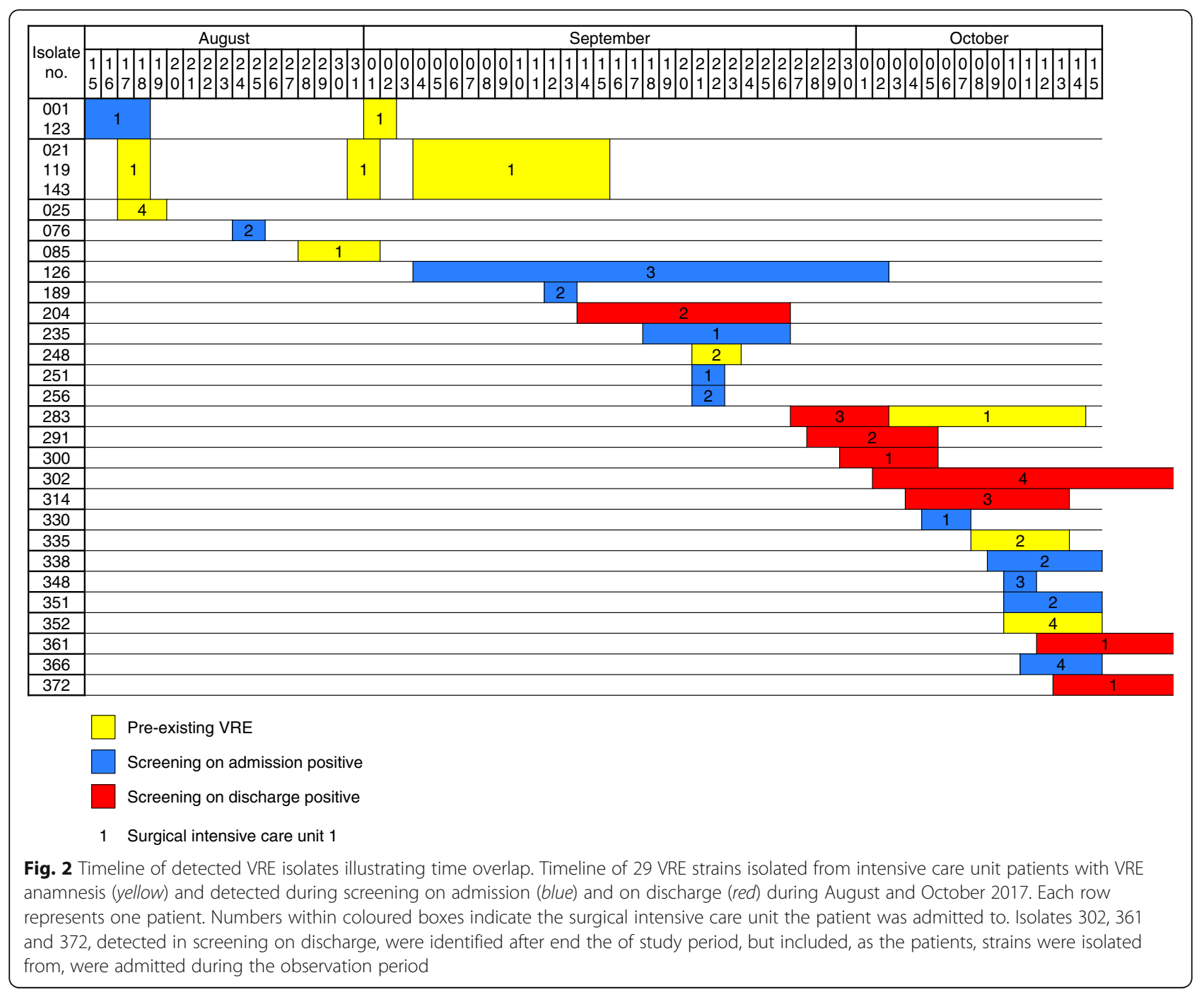

Previous contacts to VRE confirmed patients are often mentioned as a major risk factor in VRE acquisition [26]. Considering the present core genome analysis of isolates, transmission of VRE on ward could not explicitly be excluded in some cases. This may be due to the transmission of this pathogen on inanimate surfaces in advance to the final diagnosis of the VRE status. Hence in case of a confirmed VRE status, adequate basic and, if necessary, intensified hygienic measures consisting of bundle strategies including contact precautions, usage of personal protective equipment, appropriate disinfection strategies, screening of contact patients and antibiotic stewardship should be implemented to avoid transmissions as good as possible.

Our study has limitations. First, the study period was relatively short. Additional risk factors might have been revealed in case of a longer study period. Moreover, distribution of detected MLST ST in found VRE could have been different. Second, we evaluated risk factors promoting VRE carriage, e.g. flucloxacillin and piperacillin/tazobactam application, but did not investigate the pathophysiological background. Future (prospective) studies are needed to reveal underlying mechanisms. Nevertheless, our findings are in accordance with results published previously [27]. Environmental sampling was not performed during the present study, which could have uncovered the role of inanimate surfaces in VRE transmission, especially in cases of clonality of isolates. Another limitation is the problem of screening sensitivity. Here, only one rectal swab was applied on admission and on discharge respectively, which can lead underestimation of VRE prevalence. However, since screening was performed the same way on admission and on discharge, acquisition rate of VRE during SICU stay can still be assessed precisely. 
Table 3 Characteristics and risk factors of admitted surgical intensive care patients with and without hospital-acquired VRE

\begin{tabular}{|c|c|c|c|c|}
\hline Characteristic & All admitted patients $(n=374)$ & $\begin{array}{l}\text { Patients with acquired VRE } \\
(n=8)\end{array}$ & $\begin{array}{l}\text { Patients without acquired/ } \\
\text { pre-existing VRE }(n=345)\end{array}$ & $p$-value \\
\hline \multicolumn{5}{|l|}{ Demographic data } \\
\hline Median age (years) & 66 (range: 14-91) & 71.5 (range: 50-78) & 65 (range: 14-91) & 0.43 \\
\hline Male gender & $254(67.9 \%)$ & $4(50.0 \%)$ & $241(69.9 \%)$ & 0.23 \\
\hline Median duration of stay (days) & 3 (range: $1-45$ ) & 14 (range: 7-30) & 3 (range: $1-45$ ) & 0.01 \\
\hline \multicolumn{5}{|l|}{ Underlying disease/treatment } \\
\hline Haemato-oncological disease & $62(16.6 \%)$ & $1(12.5 \%)$ & $58(16.8 \%)$ & 0.74 \\
\hline Immunosuppressive disease & $71(19.0 \%)$ & $1(12.5 \%)$ & $66(19.1 \%)$ & 0.63 \\
\hline Hepatic insufficiency & $17(4.5 \%)$ & $0(0 \%)$ & $15(4.3 \%)$ & 0.55 \\
\hline Liver transplantation & $8(2.1 \%)$ & $0(0 \%)$ & $8(2.3 \%)$ & 0.66 \\
\hline Renal insufficiency & $51(13.6 \%)$ & $2(25.0 \%)$ & $41(11.9 \%)$ & 0.26 \\
\hline Long-term dialysis & $10(2.7 \%)$ & $1(12.5 \%)$ & $7(2.0 \%)$ & 0.05 \\
\hline Systemic glucocorticoid treatment & $36(9.6 \%)$ & $1(12.5 \%)$ & $32(9.2 \%)$ & 0.76 \\
\hline Antibiotic treatment & $218(58.3 \%)$ & $7(87.5 \%)$ & $207(60.0 \%)$ & 0.11 \\
\hline Ampicillin & $7(3.2 \%)$ & $0(0 \%)$ & $4(1.9 \%)$ & 0.76 \\
\hline Amoxicillin & $14(6.4 \%)$ & $0(0 \%)$ & $13(6.3 \%)$ & 0.58 \\
\hline Flucloxacillin & $20(9.2 \%)$ & $2(28.6 \%)$ & $15(7.2 \%)$ & 0.01 \\
\hline Piperacillin/tazobactam & $62(28.4 \%)$ & $4(57.1 \%)$ & $55(26.6 \%)$ & 0.01 \\
\hline Cefuroxime & $130(59.6 \%)$ & $1(14.2 \%)$ & $127(61.4 \%)$ & 0.16 \\
\hline Ceftriaxone & $7(3.2 \%)$ & $0(0 \%)$ & $3(1.4 \%)$ & 0.79 \\
\hline Meropenem & $41(18.8 \%)$ & $2(28.6 \%)$ & $36(17.4 \%)$ & 0.19 \\
\hline Clindamycin & $7(3.2 \%)$ & $0(0 \%)$ & $7(3.4 \%)$ & 0.68 \\
\hline Daptomycin & $8(3.7 \%)$ & $0(0 \%)$ & $5(2.4 \%)$ & 0.73 \\
\hline Linezolid & $3(1.4 \%)$ & $0(0 \%)$ & $3(1.4 \%)$ & 0.79 \\
\hline Rifampicin & $13(6.0 \%)$ & $0(0 \%)$ & $10(4.8 \%)$ & 0.63 \\
\hline Erythromycin & $7(3.2 \%)$ & $0(0 \%)$ & $6(2.9 \%)$ & 0.71 \\
\hline Vancomycin & $19(8.7 \%)$ & $0(0 \%)$ & $16(7.7 \%)$ & 0.53 \\
\hline Fosfomycin & $7(3.2 \%)$ & $0(0 \%)$ & $6(2.9 \%)$ & 0.71 \\
\hline Trimethoprim/Sulfamethoxazole & $6(2.8 \%)$ & $0(0 \%)$ & $6(2.9 \%)$ & 0.71 \\
\hline Metronidazole & $8(3.7 \%)$ & $0(0 \%)$ & $7(3.4 \%)$ & 0.68 \\
\hline \multicolumn{5}{|l|}{ Previous contact to healthcare system } \\
\hline Admission from a foreign hospital & $1(0.3 \%)$ & $0(0 \%)$ & $1(0.3 \%)$ & 0.88 \\
\hline Admission from a domestic hospital & $188(50.2 \%)$ & $5(62.5 \%)$ & $173(50.0 \%)$ & 0.49 \\
\hline Admission from an intensive care unit & $90(24.1 \%)$ & $4(50.0 \%)$ & $76(22.0 \%)$ & 0.06 \\
\hline
\end{tabular}

Statistical significance was declared at $p \leq 0.05$ (see italicized entries)

Table 4 Multivariate analysis: risk factors associated with VRE acquisition

\begin{tabular}{lll}
\hline Risk factors $(p \leq 0.05)$ & Odds Ratio & $95 \% \mathrm{Cl}$ \\
\hline Duration of stay & 0.90 & $0.84-0.96$ \\
Long-term-dialysis & 0.08 & $0.01-1.10$ \\
Flucloxacillin treatment & 0.09 & $0.01-0.60$ \\
Piperacillin/tazobactam treatment & 0.24 & $0.05-1.13$ \\
\hline
\end{tabular}

\section{Conclusion}

Compared to patient clienteles previously investigated [23, 28-30], SICU patients per se are not at higher risk of VRE acquisition if hygiene measures are applied as recommended. If a risk adapted screening policy is applied as practised in other national institutions [22], stay on a SICU should not be considered as a risk factor for screening upon admission to a healthcare facility.

Abbreviations

SICU: Surgical intensive care unit; VRE: Vancomycin resistant enterococci 


\section{Authors' contributions}

SK and AK: Conception and design of the study, acquisition, analysis and interpretation of data, drafting article. LMC and DK: Acquisition, analysis and interpretation of data, revising article critically. CE, AG and HF: Interpretation of data, revising article critically. AM: Conception and design of the study, interpretation of data, revising article critically. All authors have seen and approved the final version of the manuscript.

\section{Ethics approval and consent to participate}

All strategies and investigations were performed in accordance with the recommendations for cluster detections of nosocomial infections of the legally assigned institute for infection control and prevention (Robert Koch Institute). Present analysis was performed after cluster of VRE in SICU patients. Formal consent was therefore not required.

\section{Consent for publication}

Not applicable.

\section{Competing interests}

The authors declare that they have no competing interests.

\section{Publisher's Note}

Springer Nature remains neutral with regard to jurisdictional claims in published maps and institutional affiliations.

\author{
Author details \\ ${ }^{1}$ Institute of Hygiene, University Hospital Münster, Robert-Koch-Strasse 41, \\ 48149 Münster, Germany. ${ }^{2}$ Institute of Medical Microbiology, University \\ Hospital Münster, Münster, Germany. ${ }^{3}$ Department of Anaesthesiology, \\ Intensive Care and Pain Medicine, University Hospital Münster, Münster, \\ Germany.
}

Received: 4 April 2018 Accepted: 14 August 2018

Published online: 23 August 2018

\section{References}

1. European Center for Disease Prevention and Control. Data from the ECDC Surveillance Atlas - Antimicrobial resistance. https://ecdc.europa.eu/en/ antimicrobial-resistance/surveillance-and-disease-data/data-ecdc. Accessed 05 Nov 2017.

2. Kramer A, Schwebke I, Kampf G. How long do nosocomial pathogens persist on inanimate surfaces? A systematic review. BMC Infect Dis. 2006;6: 130.

3. Sample ML, Gravel D, Oxley C, Toye B, Garber G, Ramotar K. An outbreak of vancomycin-resistant enterococci in a hematology-oncology unit: control by patient cohorting and terminal cleaning of the environment. Infect Control Hosp Epidemiol. 2002;23:468-70.

4. McDermott H, Skally M, O'Rourke J, Humphreys H, Fitzgerald-Hughes D. Vancomycin-resistant enterococci (VRE) in the intensive care unit in a nonoutbreak setting: identification of potential reservoirs and epidemiological associations between patient and environmental VRE. Infect Control Hosp Epidemiol. 2018;39:40-5.

5. Pinholt M, Gumpert $H$, Bayliss S, Nielsen JB, Vorobieva V, Pedersen $M$, et al. Genomic analysis of 495 vancomycin-resistant enterococcus faecium reveals broad dissemination of a vanA plasmid in more than 19 clones from Copenhagen, Denmark. J Antimicrob Chemother. 2017;72:40-7.

6. Bender JK, Kalmbach A, Fleige C, Klare I, Fuchs S, Werner G. Population structure and acquisition of the vanB resistance determinant in German clinical isolates of Enterococcus faecium ST192. Sci Rep. 2016:6:21847.

7. Gawryszewska I, Zabicka D, Hryniewicz W, Sadowy E. Linezolid-resistant enterococci in polish hospitals: species, clonality and determinants of linezolid resistance. Eur J Clin Microbiol Infect Dis. 2017;36:1279-86.

8. DiazGranados CA, Zimmer SM, Klein M, Jernigan JA. Comparison of mortality associated with vancomycin-resistant and vancomycin-susceptible enterococcal bloodstream infections: a meta-analysis. Clin Infect Dis. 2005; 41:327-33

9. Reyes K, Bardossy AC, Zervos M. Vancomycin-Resistant Enterococci. Epidemiology, Infection Prevention, and Control Infect Dis Clin North Am. 2016;30:953-65

10. Derde LP, Cooper BS, Goossens H, Malhotra-Kumar S, Willems RJ Gniadkowski M, et al. Interventions to reduce colonisation and transmission of antimicrobial-resistant bacteria in intensive care units: an interrupted time series study and cluster randomised trial. Lancet Infect Dis. 2014:14:31-9.

11. Price CS, Paule S, Noskin GA, Peterson LR. Active surveillance reduces the incidence of vancomycin-resistant enterococcal bacteremia. Clin Infect Dis. 2003:37:921-8

12. Faron ML, Ledeboer NA, Buchan BW. Resistance mechanisms, epidemiology, and approaches to screening for vancomycin-resistant enterococcus in the health care setting. J Clin Microbiol. 2016;54:2436-47.

13. Zacharioudakis IM, Zervou FN, Ziakas PD, Rice LB, Mylonakis E. Vancomycinresistant enterococci colonization among dialysis patients: a meta-analysis of prevalence, risk factors, and significance. Am J Kidney Dis. 2015;65:88-97.

14. Papadimitriou-Olivgeris M, Drougka E, Fligou F, Kolonitsiou F, Liakopoulos A, Dodou $V$, et al. Risk factors for enterococcal infection and colonization by vancomycin-resistant enterococci in critically ill patients. Infection. 2014;42: $1013-22$

15. Ford CD, Lopansri BK, Haydoura S, Snow G, Dascomb KK, Asch J, et al. Frequency, risk factors, and outcomes of vancomycin-resistant enterococcus colonization and infection in patients with newly diagnosed acute leukemia: different patterns in patients with acute myelogenous and acute ymphoblastic leukemia. Infect Control Hosp Epidemiol. 2015;36:47-53.

16. Robert Koch Institut. Empfehlungen zur Prävention und Kontrolle von Methicillin-resistenten Staphylococcus aureus-Stämmen (MRSA) in medizinischen und pflegerischen Einrichtungen. Epi Bull. 2014;57:696-732.

17. Robert Koch Institut. Hygienemaßnahmen bei Infektionen oder Besiedlung mit multiresistenten gramnegativen Stäbchen. Epi Bull. 2012;55:1311-54.

18. Ford CD, Lopansri BK, Gazdik MA, Webb B, Snow GL, Hoda D, et al. Room contamination, patient colonization pressure, and the risk of vancomycinresistant enterococcus colonization on a unit dedicated to the treatment of hematologic malignancies and hematopoietic stem cell transplantation. Am J Infect Control. 2016;44:1110-5.

19. Mellmann A, Bletz S, Boking T, Kipp F, Becker K, Schultes A, et al. Real-time genome sequencing of resistant Bacteria provides precision infection control in an institutional setting. J Clin Microbiol. 2016:54:2874-81.

20. de Been M, Pinholt M, Top J, Bletz S, Mellmann A, van Schaik W, et al. Core genome multilocus sequence typing scheme for high- resolution typing of Enterococcus faecium. J Clin Microbiol. 2015;53:3788-97.

21. Robert Koch Institut. Eigenschaften, Häufigkeit und Verbreitung von Vancomycin-resistenten Enterokokken (VRE) in Deutschland - Update 2015/ 2016. Epi Bull. 2017:46:519-27.

22. Remschmidt C, Behnke M, Kola A, Pena Diaz LA, Rohde AM, Gastmeier P, et al. The effect of antibiotic use on prevalence of nosocomial vancomycinresistant enterococci- an ecologic study. Antimicrob Resist Infect Control. 2017;6:95

23. D'Agata EM, Green WK, Schulman G, Li H, Tang YW, Schaffner W. Vancomycin-resistant enterococci among chronic hemodialysis patients: a prospective study of acquisition. Clin Infect Dis. 2001;32:23-9.

24. Pan SC, Wang JT, Chen YC, Chang YY, Chen ML, Chang SC. Incidence of and risk factors for infection or colonization of vancomycin-resistant enterococci in patients in the intensive care unit. PLoS One. 2012;7:e47297.

25. Hensgens MP, Goorhuis A, Dekkers OM, Kuijper EJ. Time interval of increased risk for Clostridium difficile infection after exposure to antibiotics. J Antimicrob Chemother. 2012;67:742-8.

26. Tacconelli E, Cataldo MA. Vancomycin-resistant enterococci (VRE): transmission and control. Int J Antimicrob Agents. 2008:31:99-106.

27. Karki S, Land G, Aitchison S, Kennon J, Johnson PD, Ballard SA, et al. Longterm carriage of vancomycin-resistant enterococci in patients discharged from hospitals: a 12-year retrospective cohort study. J Clin Microbiol. 2013; 51:3374-9.

28. Vydra J, Shanley RM, George I, Ustun C, Smith AR, Weisdorf DJ, et al. Enterococcal bacteremia is associated with increased risk of mortality in recipients of allogeneic hematopoietic stem cell transplantation. Clin Infect Dis. 2012;55:764-70

29. Flokas ME, Karageorgos SA, Detsis M, Alevizakos M, Mylonakis E. Vancomycin-resistant enterococci colonisation, risk factors and risk for infection among hospitalised paediatric patients: a systematic review and meta-analysis. Int J Antimicrob Agents. 2017;49:565-72.

30. Ziakas PD, Pliakos EE, Zervou FN, Knoll BM, Rice LB, Mylonakis E. MRSA and VRE colonization in solid organ transplantation: a meta-analysis of published studies. Am J Transplant. 2014;14:1887-94. 